\title{
Response to Correspondence to Hale et al. Atypical Phenotypes Associated with Pathogenic CHD? Variants and a Proposal for Broadening CHARGE Syndrome Clinical Diagnostic Criteria
}

\author{
Caitlin L. Hale, ${ }^{1}$ Adrienne N. Niederriter, ${ }^{2}$ Glenn E. Green, ${ }^{3}$ and Donna M. Martin ${ }^{2,4,5 *}$ \\ ${ }^{1}$ Department of Pediatrics, Stanford University, Stanford, California \\ ${ }^{2}$ Medical Scientist Training Program, The University of Michigan Medical School, Ann Arbor, Michigan \\ ${ }^{3}$ Department of Otolaryngology, The University of Michigan Medical School, Ann Arbor, Michigan \\ ${ }^{4}$ Department of Human Genetics, The University of Michigan Medical School, Ann Arbor, Michigan \\ ${ }^{5}$ Department of Pediatrics and Communicable Diseases, The University of Michigan Medical School, Ann Arbor, Michigan
}

Manuscript Received: 2 February 2016; Manuscript Accepted: 26 February 2016

\section{TO THE EDITOR:}

We appreciate the comments by Blake et al. [2016] regarding our manuscript "Atypical phenotypes associated with pathogenic CHD7 sequence variants and a proposal for broadening CHARGE syndrome clinical diagnostic criteria" [Hale et al., 2016]. Blake et al. emphasize (as we do in our paper) that clinicians need to be aware of the wide variety of clinical presentations associated with CHARGE syndrome. They also question the appropriateness of our minor criteria and how they influence clinical diagnosis and care. We welcome these questions and provide clarification in order to promote discussion and to ensure that these proposed criteria are accessible to clinicians outside the genetics community.

The most recent clinical diagnostic criteria for CHARGE, established by Verloes [2005], were robust and comprehensive, and allowed for wide variability in clinical presentation [Verloes, 2005]. The Verloes criteria built upon earlier criteria by Blake et al. [1998], and included the same major criteria as we propose in our manuscript. In contrast to earlier diagnostic criteria (and perhaps a source of confusion), our proposed "minor criteria" are better thought of as associated features, since their presence or absence does not influence the final diagnosis of CHARGE syndrome. For example, "cranial nerve (CN) dysfunction" is a minor criterion since it encompasses dysfunction of cranial nerves other than coloboma (CN-II) or hearing loss (CN-VIII), both of which are major criteria. Manifestations of other CN dysfunction (e.g., CNs-V, VII, IX, and X), including dysphagia, and anosmia/hyposmia (CN-I) are very common among individuals with CHARGE. Abnormalities of $\mathrm{CN}$ function are also common among children with other causes of growth and/or developmental delay, and are not specific to CHARGE. "Mental retardation" in Verloes' criteria was expanded in our proposal to "developmental delay (DD)/ intellectual disability (ID)/autism spectrum disorder (ASD),"

\section{How to Cite this Article:}

Hale CL, Niederriter AN, Green GE, Martin DM. 2016. Response to correspondence to Hale et al. atypical phenotypes associated with pathogenic $\mathrm{CHD} 7$ variants and a proposal for broadening CHARGE syndrome clinical diagnostic criteria. Am J Med Genet Part A 170A:3367-3368.

recognizing that these are clinical diagnoses whose presentation and severity varies widely across individuals and whose assessments are complicated when sensory impairments are present.

Hypothalamic-hypophyseal dysfunction is also a common feature in CHARGE, and occurs with Kallmann syndrome. Since both CHARGE and Kallmann syndromes are associated with CHD7 pathogenic variants, an individual with a pathogenic CHD7variant and hypothalamic-hypophyseal dysfunction should be clinically evaluated for other features of CHARGE, as pointed out by others [Jongmans et al., 2009] and discussed in our paper.

It can be challenging to establish diagnostic criteria for genetic conditions that present with broad phenotypic heterogeneity. In the era before molecular testing for $\mathrm{CHD} 7$ variants in CHARGE syndrome (i.e., prior to 2004), clinicians relied exclusively

\footnotetext{
*Correspondence to:

Donna M. Martin, M.D., Ph.D, Department of Pediatrics and Communicable Diseases and Human Genetics, The University of Michigan Medical School, 3520A MSRBI, 1150 Medical Center Drive, SPC 5652, Ann Arbor, MI 48109-5652.

E-mail: donnamm@umich.edu

Article first published online in Wiley Online Library

(wileyonlinelibrary.com): 21 March 2016

DOI 10.1002/ajmg.a.37629
} 
on the presence or absence of specific features to establish the diagnosis. It is now possible, however, to clarify whether or not a sequence variant in $\mathrm{CHD} 7$ should be considered pathogenic for the underlying features. There are specific (and extremely useful) guidelines for when to test for $\mathrm{CHD} 7$ sequence variants in individuals with CHARGE features [Bergman et al., 2011]. Accurate and meaningful genetic information can lead to improved understanding of etiology, provide accurate recurrence risks, and help pave the way toward better clinical care. We advocate incorporating $\mathrm{CHD} 7$ sequence variant information into the diagnostic algorithm, when it is available, since this information can improve understanding of disease causation, pathogenesis, and treatment options. In cases when CHD7 variant testing is not available, the diagnosis can still be made based on appropriate clinical assessments.

The American College of Medical Genetics and Genomics (ACMG) recognizes the complexities of and proposes guidelines for evaluating potential pathogenicity of sequence variants [Richards et al., 2015]. Disease-specific databases (e.g., www.chd7.org), population frequency, functional and expression data, phenotype-genotype correlations, review of published literature, and review of databases such as Exome Aggregation Consortium (ExAC), 1000 Genomes, and the Exome Variant Server from the NHLBI Exome Sequencing Project (ESP) are all critical to accurate pathogenicity assignment. This discourse about the appropriateness of a combined clinical and molecular diagnosis for CHARGE is therefore both relevant and timely.

\section{REFERENCES}

Bergman JEH, Janssen N, Hoefsloot LH, Jongmans MCJ, Hofstra RMW, van Ravenswaaij-Arts CMA. 2011. CHD7 mutations and CHARGE syndrome: The clinical implications of an expanding phenotype. J Med Genet 48:334-342.

Blake K, Trider C, Hartshorne TS, Stratton KK. 2016. Correspondence to hale et al. atypical phenotypes associated with pathogenic CHD7 variants and a proposal for broadening CHARGE syndrome clinical diagnostic criteria. Am J Med Genet Part A 170A (this issue).

Blake KD, Davenport SL, Hall BD, Hefner MA, Pagon RA, Williams MS, Lin AE, Graham JM, Jr. 1998. CHARGE association: An update and review for the primary pediatrician. Clin Pediatr 37:159-173.

Hale CL, Niederriter AN, Green GE, Martin DM. 2016. Atypical phenotypes associated with pathogenic CHD7 variants and a proposal for broadening CHARGE syndrome clinical diagnostic criteria. Am J Med Genet Part A 170A:344-354.

Jongmans MCJ, van Ravenswaaij-Arts CMA, Pitteloud N, Ogata T, Sato N, Claahsen-van der Grinten HL, van der Donk K, Seminara S, Bergman JEH, Brunner HG, Crowley WF, Hoefsloot LH. 2009. CHD7 mutations in patients initially diagnosed with Kallmann syndrome-The clinical overlap with CHARGE syndrome. Clin Genet 75:65-71.

Richards S, Aziz N, Bale S, Bick D, Das S, Gastier-Foster J, Grody WW, Hegde M, Lyon E, Spector E, Voelkerding K, Rehm HL, Committee ALQA. 2015. Standards and guidelines for the interpretation of sequence variants: A joint consensus recommendation of the American College of Medical Genetics and Genomics and the Association for Molecular Pathology. Genet Med 17:405-424.

Verloes A. 2005. Updated diagnostic criteria for CHARGE syndrome: A proposal. Am J Med Genet Part A 133A:306-308. 